\title{
Comparação entre a avaliação da amplitude articular estática do cotovelo por meio de três diferentes métodos: goniometria, biofotogrametria e goniometria da imagem radiológica Comparison of evaluation of static elbow range of motion using three different methods: goniometry, biophotogrammetry and radiological goniometry
}

Rodrigo Luis Ferreira da Silva, Ft.*, Renato Ramos Coelho, Ft., M.Sc.**, Gabriela Amorim Barreto, Ft.***, Janaína Portela de Aguiar, Ft. ${ }^{* *}$, Pedro Odimar dos Santos, Ft. ${ }^{* * *}$, Estélio H. M. Dantas, D.Sc. ${ }^{* * * *}$

*Mestrando em ciência da motricidade humana pela UCB/RJ, Professor efetivo da UEPA, **Professor da UNIPAC Bom Despacho, ${ }^{* * *}$ Curso de Fisioterapia da UEPA, ${ }^{* * *}$ Mestrando em ciência da motricidade humana pela UCB/RJ, Professor da UEPA, ****Orientador do mestrado em ciência da motricidade humana da UCB/RJ, bolsista de produção cientifi ca do CNPQ

\section{Resumo}

Introdução: $\mathrm{O}$ objetivo deste estudo foi verificar a confiabilidade e objetividade da goniometria e da biofotogrametria para a avaliação de uma posição do cotovelo através da comparação dos dados obtidos por estes métodos com os obtidos pela goniometria radiológica. Material e Métodos: A pesquisa contou com dezesseis indivíduos, sendo seis examinadores e dez avaliados. Para todas as técnicas de medida utilizadas, cada avaliador colheu três medidas de um mesmo ângulo do cotovelo (143ㅇ), que foi considerado como ângulo alvo das análises estatísticas e neste estudo chamado de ângulo chave. Resultados: Após a análise estatística, verificou-se baixa e moderada confiabilidade para a goniometria, e alta e muito alta confiabilidade para a biofotogrametria. Quanto à objetividade, verificou-se valores alto e moderado com o uso do goniômetro, ao passo que com a biofotogrametria somente se observaram valores altos. No entanto, as medidas encontradas com essas técnicas não apresentaram boa correlação com os valores obtidos pela goniometria radiológica. Conclusão: A técnica biofotogramétrica apresentou maior confiabilidade e objetividade em comparação com a goniometria sem, no entanto, apresentar correlaçáo de seus dados com os obtidos pela goniometria radiológica.

Palavras-chave: goniometria, biofotogrametria, goniometria radiológica.

\begin{abstract}
Introduction: The aim of this study was to verify the reliability and objectivity of goniometry and biophotogrammetry to evaluate one specific elbow position. Data obtained from these two methods were compared with radiological goniometry data. Material \& $\mathrm{Me}$ thods: The group was composed by sixteen subjects: six examiners and ten volunteers. For all measurements used in this research each examiner took three measures of same angle of the elbow $\left(143^{\circ}\right)$ which was considered as target angle of statistical analyses in this study called key angle. Results: After statistical analysis, it was checked low and moderated reliability to the goniometry and high and very high reliability to biophotogrammetry. In regards to objectivity, it was observed higher and moderate reliability with the goniometer while with the biophotogrammetry only higher values. Though, measurements found with these techniques did not show good correlation with radiological goniometry values. Conclusion: The biophotogrammetry technique showed more reliability and objectivity in comparison to goniometry, however did not show correlation with radiological goniometry data values.
\end{abstract}

Key-words: goniometry, biophotogrammetry, radiological goniometry. 


\section{I ntrodução}

A tomada de medida da amplitude de movimento articular (ADM) é uma prática essencial na avaliação fisioterapêutica, pois ajuda a identificar possíveis limitaçôes articulares, definindo muitas vezes o tratamento e o prognóstico de um indivíduo submetido à Fisioterapia, bem como permite a estes profissionais acompanharem de modo quantitativo a eficácia das intervençóes terapêuticas [1,2].

Esta necessidade clínica de se mensurar a ADM trouxe significativas evoluções quanto às técnicas de sua medição [3]. Isto fez surgir um número bastante expressivo de métodos e instrumentos capazes de avaliar os graus do deslocamento angular que se processam nas articulaçóes, cada qual com sua vantagem, desvantagem e técnica de aplicabilidade [4-8].

Até os dias atuais, o instrumento mais utilizado para medida de ADM é o goniômetro universal [3,4], já existindo diversos estudos que comprovam sua validade quando comparada à goniometria radiológica, considerada como padrão ouro para este tipo de avaliação [4,9].

Este instrumento, no entanto, requer uma técnica bastante precisa de localização, palpação e alinhamento dos braços do goniômetro com referências ósseas já padronizadas $[4,10]$. Esse fato dificulta a realização de avaliaçôes goniométricas em, por exemplo, indivíduos obesos, em que há uma maior dificuldade para a palpação e localização das referências ósseas. Outra limitação é a considerável experiência exigida pelo examinador [11].

Instrumentos como o flexímetro e o inclinômetro, por sua vez, não necessitam da realização de palpaçóes ou alinhamentos, no entanto, exigem um rigor bastante elevado no que se refere ao posicionamento do avaliado, principalmente quando se faz necessária a comparação de avaliaçôes que foram realizadas em dias diferentes $[4,5,12]$.

Isto ocorre por que estes instrumentos baseiam-se no princípio da atraçáo gravitacional, no qual a mínima alteração do posicionamento do segmento avaliado pode colocar o instrumento em uma posição espacial diferente da original, recebendo uma ação diferente da força gravitacional, e, portanto produzindo, ao final, valores muito diferentes do apresentado pela articulação avaliada $[4,5,12]$.

A evolução tecnológica surge como possibilidade de dinamizar a mensuração do movimento articular, preservando a confiabilidade em suas medidas, através de novas técnicas e instrumentos, mais práticos e sofisticados para este fim. Um exemplo é o da biofotogrametria que já é bastante difundida no meio fisioterapêutico [3,13-15].

No entanto, este grande número de possibilidades de instrumentos e técnicas de avaliação da ADM merece uma reflexão por parte dos profissionais fisioterapeutas que utilizam estes recursos, no sentido de não se deixarem levar simplesmente pela facilidade de seu uso, em prejuízo da reprodutibilidade dos valores que ele expressa.
Diz-se que uma medida é reprodutível se provém de um procedimento reprodutível, ou seja, se repetido nas mesmas condiçōes obtêm-se resultados semelhantes. A falta de reprodutibilidade nas medidas pode levar a importantes conseqüências ao nível científico e clínico [19]. Para Portney [16] a reprodutibilidade das medidas demonstra a consistência obtida por um instrumento ou por um examinador nas mesmas condiçóes de avaliaçấo e é dividida em dois aspectos diferentes e complementares. A confiabilidade é a consistência das medidas realizadas nas mesmas condições de avaliação em momentos diferentes. Já a objetividade vincula-se à consistência das medidas realizadas por examinadores diferentes [18].

A confiabilidade e a objetividade de uma medida são, portanto, essenciais para garantir a consistência dos dados, possibilitando sua utilização em pesquisas científicas e a evolução dos tratamentos na clínica fisioterapêutica. Essa consistência de resultados representa a reprodutibilidade, ou seja, o excelente grau de concordância tanto para confiabilidade, quanto para objetividade [16-18].

Este estudo teve o objetivo de analisar a confiabilidade e a objetividade de duas destas técnicas de medida de ADM, comparando-as com a goniometria radiológica, tida como o padrão ouro para este tipo de avaliação [20], identificando o instrumento mais fidedigno para a medida de movimentação articular.

\section{Material e métodos}

Selecionou-se uma amostra de dez (10) indivíduos maiores de 18 anos de idade, do gênero masculino, com idade entre 18 a 26 anos $( \pm 21,3$ anos e $S D=2,62)$, sem histórico de patologias ortopédicas e/ou neurológicas que interferissem na mobilidade e anatomia normal dos ossos no membro superior direito (MSD). Eles também não apresentavam nenhuma contra-indicação para a exposição aos raios X. Seis (06) examinadores foram selecionados, sendo cinco (05) fisioterapeutas e professores do curso de fisioterapia da UEPA/STM, e um (01) acadêmico do último ano $\left(5^{\circ}\right)$ do curso de fisioterapia da UEPA/STM. Entre os fisioterapeutas a experiência profissional variou de quatro (04) a quatorze (14) anos.

A tomada de medida dos ângulos foi realizada com os avaliados, que tiveram a sua articulação do cotovelo direito estabilizada por chapas de ferro retorcido que possuíam, entre si, ângulos diversos. Dentre estes ângulos apenas um repetiase por três vezes e serviu como o ângulo chave da pesquisa $\left(143^{\circ}\right)$. O cotovelo foi estabilizado em máxima supinação para todas as avaliaçôes de ADM, independentemente do instrumento de medida utilizado. Em nenhum momento os avaliadores tiveram acesso ao valor do ângulo existente entre as chapas de ferro retorcido.

As mensuraçôes ocorreram em três momentos distintos:

1) Primeiramente foram realizadas as avaliaçóes de ADM, com o instrumento goniômetro. Os examinadores mediram por três vezes, cada um, os ângulos das articulaçóes dos cotove- 
los dos avaliados. Estas medidas foram feitas em um único dia com os examinadores realizando rodadas alternadas de avaliação, em um grupo de avaliados que mesclava outros ângulos, além do ângulo chave $\left(143^{\circ}\right)$. Esta conduta foi tomada para evitar que os avaliadores percebessem qual dos ângulos seria o ângulo chave.

2) Três dias após este primeiro momento, foi realizada a captura das imagens digitais para a mensuração dos ângulos através da biofotogrametria. Os avaliados mantinham-se em posição ortostática, e cada avaliador palpava as referências ósseas e as marcava com adesivos coloridos que identificavam cada um dos avaliadores. As referências ósseas padronizadas foram as seguintes: epicôndilo lateral do úmero, processo estilóide do rádio, e centro articular lateral da glenoumeral que segundo Field [21] localiza-se aproximadamente a $2 \mathrm{~cm}$ abaixo da metade anterior da margem lateral do acrômio. Da mesma forma como a utilizada para a goniometria, cada um dos avaliadores realizou três seqüências de palpação e demarcação em cada um dos avaliados.

A captura da imagem ocorreu com câmera fotográfica digital, marca Sony - 6.0 megapixels, com lente Carl Zeiss posicionada a uma distância de dois metros do voluntário a ser fotografado, na altura da articulaçáo do cotovelo de cada indivíduo, a partir do solo. As fotografias obtidas foram então calibradas através do software GML Camera Calibration (Departamento de Ciência da Computação da Universidade Estadual de Moscou, Rússia), e em seguida analisadas pelo software AutoCad 2005(AutoDesk Inc. EUA) para a verificação do ângulo obtido [22].

3) Por fim, os avaliados tiveram o seu membro superior direito radiografado, com o foco sobre a articulação do cotovelo fixada pela chapa de ferro do ângulo chave (1430) e sendo realizada uma única radiografia desta posição angular. Este procedimento foi executado por um profissional técnico em radiologia que orientou o posicionamento dos avaliados. Os ângulos das radiografias foram obtidos através do protocolo descrito por Dantas et al. [20], traçando-se retas correspondentes às bissecções das diáfises dos ossos úmero e rádio. Posteriormente, utilizou-se um goniômetro metálico, tamanho grande da marca Baseline Stainless, para a leitura dos ângulos obtidos.

Figura 1 - Avaliação Biofotogramétrica do cotovelo pelo AutoCad 2005.

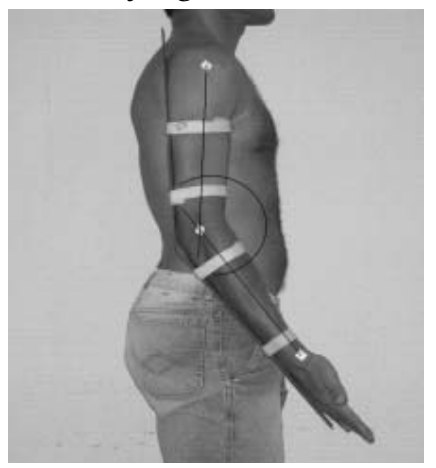

Figura 2 - Radiografia do cotovelo para goniometria radiológica.

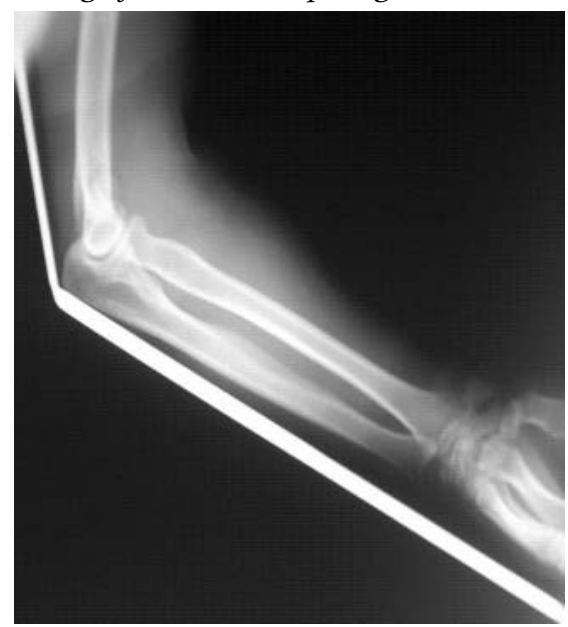

Os valores dos ângulos colhidos receberam tratamento estatístico através do software Statistical Package for Social Science 14.0 (SPSS Inc, USA), com o objetivo de determinar a normalidade de sua distribuição, a reprodutibilidade destes dados e a existência, ou não, de correlação entre os dados obtidos pelas avaliaçóes realizadas com as diferentes técnicas e instrumentos.

Após análise descritiva dos dados, a confiabilidade da ADM estática do cotovelo, tomada pelas diferentes técnicas, foi determinada através do cálculo do Coeficiente de Correlação Intraclasse (CCI) tipo 3,1 $\left(\mathrm{CCI}_{(3,1)}\right)$. Para o cálculo da objetividade entre as avaliaçóes dos diferentes avaliados, foi utilizado o cálculo do Coeficiente de Correlação Intraclasse tipo 2,3 $\left(\mathrm{CCI}_{(2,3)}\right)$. Neste estudo, os valores de CCI foram considerados baixo quando menores que 0.50 , moderado entre 0.50 a 0.75 , alto acima de 0.75 e muito alto quando superiores a 0.90, segundo o descrito por Portney et al. [16].

Para determinar a correlação entre os valores obtidos pela goniometria e pela biofotogrametria, com os obtidos pela goniometria radiológica, adotou-se o teste de Pearson, enquanto que o teste t pareado foi empregado para avaliar a estabilidade dos valores obtidos pelas diferentes técnicas. Adotou-se uma significância de $\mathrm{p}<0,05$.

\section{Resultados}

Os dados da confiabilidade para as duas técnicas de medida de ADM, envolvidas nesta pesquisa, estão apresentadas nas tabelas I e II, demonstrando perceptível vantagem para a biofotogrametria nos valores encontrados pelos avaliadores.

A Tabela I demonstra que entre os seis (06) avaliadores que mediram o ângulo chave $\left(143^{\circ}\right)$ com o goniômetro, dois (02) deles (A e C) apresentaram valores baixos de confiabilidade, ao passo que os outros quatro (04) avaliadores, alcançaram valores apenas moderados para confiabilidade de suas medidas com o goniômetro. 
Tabela I - Confiabilidade das medidas de seis avaliadores para as ADM de um ângulo fixo da articulação do cotovelo de 10 individuos, como o uso de goniômetro.

\begin{tabular}{lllll}
\hline \multicolumn{5}{l}{ Confiabilidade Goniômetro } \\
\hline \multicolumn{5}{c}{ Intervalo de confiança de 95\% } \\
\cline { 2 - 5 } & $\mathrm{CCl}(3,1)$ & limite inferior & limite superior & $\mathrm{p}$ \\
\hline A & 0,26 & $-0,13$ & 0,69 & 0,11 \\
B & 0,58 & 0,23 & 0,86 & 0,00 \\
C & 0,26 & $-0,13$ & 0,70 & 0,10 \\
D & 0,53 & 0,15 & 0,84 & 0,00 \\
E & 0,73 & 0,42 & 0,91 & 0,00 \\
F & 0,52 & 0,15 & 0,83 & 0,00 \\
\hline
\end{tabular}

CCl: Coeficiente de Correlação Intraclasse; p: Nível descritivo do teste de significância estatística.

$\mathrm{Na}$ Tabela II observa-se que somente um dos avaliadores (F) alcançou valor de alta confiabilidade, enquanto que os demais avaliadores obtiveram valores de muito alta confiabilidade para as sua medidas do ângulo chave $\left(143^{\circ}\right)$ com o uso da biofotogrametria.

Tabela II - Confiabilidade das medidas de seis avaliadores para as $A D M$ de um ângulo fixo da articulação do cotovelo de 10 indivíduos, como o uso da biofotogrametria.

\begin{tabular}{lllll}
\multicolumn{5}{l}{ Confiabilidade } \\
\hline \multicolumn{5}{c}{ Biofotogrametria } \\
\cline { 2 - 5 } & $\mathrm{CCl}(3,1)$ & limite inferior & limite superior & $\mathrm{p}$ \\
\hline A & 0,96 & 0,90 & 0,99 & 0,00 \\
B & 0,93 & 0,82 & 0,98 & 0,00 \\
C & 0,94 & 0,85 & 0,98 & 0,00 \\
D & 0,93 & 0,80 & 0,98 & 0,00 \\
E & 0,94 & 0,83 & 0,99 & 0,00 \\
F & 0,83 & 0,60 & 0,95 & 0,00 \\
\hline
\end{tabular}

CCl: Coeficiente de Correlação Intraclasse; p: Nível descritivo do teste de significância estatística.

Os dados referentes à avaliação da objetividade para as duas técnicas de medida da ADM, envolvidas nesta pesquisa estão apresentados nas Tabelas III e IV. Os resultados observados demonstraram que com o goniômetro as medidas continuaram apresentando desvantagem (menor objetividade) em relação às medidas com a biofotogrametria, no entanto, demonstrando agora uma menor disparidade entre as técnicas.

$\mathrm{Na}$ Tabela III observa-se que, com o uso da goniometria, os avaliadores alcançaram valores altos de objetividade para as suas duas primeiras rodadas de avaliaçáo do ângulo chave $\left(143^{\circ}\right)$, e valor moderado de objetividade para a terceira rodada de medidas.
Tabela III - Objetividade das medidas de seis avaliadores, para as $A D M$ de um ângulo fixo da articulação do cotovelo de 10 indivíduos, como o uso de goniômetro.

\section{Objetividade Goniômetro}

\begin{tabular}{lllll}
\hline & \multicolumn{4}{c}{ Intervalo de confiança de 95\% } \\
\cline { 2 - 5 } & $\mathbf{C C l}$ & limite & limite \\
& $(\mathbf{2 , 3})$ & inferior & superior & $\mathbf{p}$ \\
\hline Média 1 medida & 0,87 & 0,68 & 0,96 & 0,00 \\
Média 2 medida & 0,83 & 0,60 & 0,95 & 0,00 \\
Média 3 medida & 0,73 & 0,35 & 0,92 & 0,00 \\
\hline CCl: Coeficiente de Correlação Intraclasse; p: Nível descritivo do teste \\
de significância estatística.
\end{tabular}

A Tabela IV demonstra que com o uso da biofotogrametria os avaliadores foram mais consistentes em suas medidas, alcançando somente valores altos de objetividade para as suas três rodadas de avaliação do ângulo chave (143).

Tabela IV - Objetividade das medidas de seis avaliadores, para as $A D M$ de um ângulo fixo da articulação do cotovelo de 10 individuos, como o uso da biofotogrametria.

\begin{tabular}{lllll}
\hline Objetividade Biofotogrametria \\
\cline { 2 - 5 } & \multicolumn{4}{c}{ Intervalo de confiança de $95 \%$} \\
\hline & $\mathbf{C C l}$ & limite & limite \\
& $(\mathbf{2 , 3})$ & inferior & superior & $\mathbf{p}$ \\
\hline média 1 medida & 0,89 & 0,74 & 0,97 & 0,00 \\
média 2 medida & 0,86 & 0,67 & 0,96 & 0,00 \\
média 3 medida & 0,90 & 0,75 & 0,97 & 0,00 \\
\hline
\end{tabular}

CCl: Coeficiente de Correlação Intraclasse; p: Nível descritivo do teste de significância estatística.

As Tabelas V e VII demonstram o grau de correlação entre os valores médios das medidas obtidas pela goniometria e pela biofotogrametria, com valores médios das medidas obtidas pela goniometria radiológica, empregando o teste de Pearson.

As Tabelas VI e VIII, por sua vez, demonstram a estabilidade dos valores obtidos pelas diferentes técnicas, avaliados pelo teste $\mathrm{t}$ pareado.

Tabela V - Valor da correlação entre a média das medidas obtidas pela goniometria radiológica e a média das medidas obtidas pela goniometria.

\begin{tabular}{llllllll}
\hline \multicolumn{3}{c}{ Estatística descritiva } & \multicolumn{3}{c}{ Correlação de Pearson } \\
\hline & Média & SD & N & $\begin{array}{l}\text { Radio- } \\
\text { grafia }\end{array}$ & $\begin{array}{l}\text { Gonio- } \\
\text { metria }\end{array}$ & P \\
\hline GON & & & & & 0,619 & 0,057 \\
RAD & 142 & 6,72 & 10 & 1 & 0,619 & 1 & 0,057 \\
GON & 140,12 & 5,05 & 180 & 0,619 &
\end{tabular}

A Tabela $\mathrm{V}$ demonstrou a existência de uma correlação de 0,619, tida como moderada, entre as medidas obtidas pela goniometria radiológica e pela goniometria, para um valor de $p<0,057$. 
Tabela VI - Resultados da comparação entre os dados obtidos pela goniometria radiológica e os obtidos pela goniometria pelo teste $t$.

\begin{tabular}{|c|c|c|c|c|c|c|c|c|}
\hline & \multirow[b]{2}{*}{ Média } & \multirow[b]{2}{*}{ SD } & \multirow[b]{2}{*}{ SE } & \multicolumn{2}{|c|}{ IC 95\% } & \multirow[b]{2}{*}{ t } & \multirow[b]{2}{*}{ Df } & \multirow[b]{2}{*}{ P } \\
\hline & & & & Menor & Maior & & & \\
\hline \multicolumn{9}{|l|}{ RAD - } \\
\hline GON & 4,2 & 5,514 & 1,744 & 0,256 & 8,144 & 2,409 & 9 & 0,039 \\
\hline
\end{tabular}

O teste $t$ para amostras pareadas, por sua vez, indicou que houve diferença estatística entre as médias dos dados obtidos pela goniometria radiológica e pela goniometria $(p=0,039)$, conforme pode ser observado na Tabela VI.

Tabela VII - Valor da correlação entre a média das medidas obtidas pela goniometria radiológica e a média das medidas obtidas pela biofotogrametria.

\begin{tabular}{llllllll}
\hline \multicolumn{4}{c}{ Estatística descritiva } & \multicolumn{3}{c}{ Correlação de Pearson } \\
\hline & Média & SD & N & $\begin{array}{l}\text { Radio- } \\
\text { grafia }\end{array}$ & $\begin{array}{l}\text { Biofoto- } \\
\text { grametria }\end{array}$ \\
\hline GON & & & & & 0,268 & 0,454 \\
RAD & 142 & 6,72 & 10 & 1 & 0,268 & 1 & 0,454 \\
BIOF & 137,09 & 5,56 & 180 & 0,268 & 1
\end{tabular}

A Tabela VII demonstrou a existência de uma correlação de 0,268 , tida como baixa, entre as medidas obtidas pela goniometria radiológica e pela biofotogrametria, para um valor de $p<0,454$.

Tabela VIII - Resultados da comparação entre os dados obtidos pela goniometria radiológica e os obtidos pela biofotogrametria pelo teste $t$.

\begin{tabular}{|c|c|c|c|c|c|c|c|c|}
\hline & \multirow[b]{2}{*}{ Média } & \multirow[b]{2}{*}{ SD } & \multirow[b]{2}{*}{ SE } & \multicolumn{2}{|c|}{ IC 95\% } & \multirow[b]{2}{*}{$t$} & \multirow[b]{2}{*}{ Df } & \multirow[b]{2}{*}{$P$} \\
\hline & & & & Menor & Maior & & & \\
\hline \multicolumn{9}{|c|}{ RAD - } \\
\hline $\mathrm{BIOF}$ & 7,7 & 7,334 & 2,319 & 2,454 & 12,946 & 3,32 & 9 & 0,009 \\
\hline
\end{tabular}

A Tabela VIII, por sua vez, indicou que houve diferença estatística entre as médias dos dados obtidos pela goniometria radiológica e pela biofotogrametria $(p=0,009)$, conforme o teste $t$ para amostras pareadas.

Portanto, os resultados das mediçóes, quando analisados estatisticamente, revelam que tanto a goniometria quanto a biofotogrametria não apresentaram boa correlaçáo com os valores obtidos pela goniometria radilógia, que é considerada como referência de amplitude real. Observa-se ainda baixa estabilidade dos dados alcançados com as duas primeiras técnicas, demonstrando que os valores verificados pelos avaliadores são estatisticamente diferentes para estas técnicas.

\section{Discussão}

Os resultados deste trabalho apresentaram baixa e moderada confiabilidade para os valores obtidos pela goniometria. Vários estudos comparativos entre técnicas de medida de amplitude articular, como os de Venturini et al. [1] e Tiziani et al. [23], já demonstraram este mesmo comportamento dos resultados. No entanto, devido à diversidade metodológica entre os trabalhos, torna-se difícil discutir a relação de seus resultados com os deste estudo.

Venturini et al. [1] trabalhou com um n de 22 indivíduos entre homens e mulheres, realizando a avaliaçáo de ADM ativa de dorsiflexão de tornozelo, tanto em cadeia cinética aberta quanto em cadeia cinética fechada. As medidas em cadeia cinética aberta foram realizadas com o uso de um goniômetro universal, enquanto que para as medidas em cadeia cinética fechada os voluntários posicionavam o pé sobre uma fita métrica, realizando o movimento de dorsiflexão do tornozelo em direçáo posterior em relação a uma parede, até o máximo possível. As avaliações deste estudo foram executadas por dois avaliadores, que realizaram três medidas com cada uma das técnicas de avaliação, um imediatamente ao outro.

O estudo descrito acima observou melhores resultados de confiabilidade e objetividade para as medidas realizadas em cadeia cinética fechada, sugerindo que esse método seja mais confiável para a aplicação clínica que envolva um ou mais examinadores durante o acompanhamento fisioterapêutico.

Tiziani et al. [23] realizou sua pesquisa com 30 indivíduos, entre homens e mulheres, realizando avaliação ativa de flexão e extensão de punho, com o uso da biofotogrametria, analisada pelo programa Coreldraw 12, e da goniometria realizada três vezes consecutivas pelo mesmo avaliador. Em seus resultados este estudo não demonstrou validade concorrente entre os dois métodos.

De maneira geral, estes estudos têm demonstrado melhores valores de confiabilidade quando as sessôes de avaliação são executadas em um pequeno intervalo de tempo, considerado como menor de 15 minutos [1]. Contudo, a discussão sobre este tema parece ainda estar muito distante de seu desfecho, haja vista que também já foram encontrados valores de boa confiabilidade com o uso do goniômetro em pesquisas com intervalo de tempo superior a 48 horas entre as avaliaçóes $[12,24]$.

Pode-se supor que a obstrução visual do alinhamento do eixo articular, ocorrida pela utilização de um goniômetro metálico neste estudo, pode ter colaborado para estes resultados negativos. Tal fato já foi percebido por Norkin et al. [4], em uma análise sobre um estudo de Fish et al. [25].

No caso específico deste estudo, a maratona de rodadas alternadas para a tomada destas medidas pode ter desgastado os avaliadores em questáo, resultando na imprecisão de suas medidas, o que pode assim caracterizar um viés de coleta de dados [26].

Um fato que reforça esta hipótese deve-se ao excelente nível de confiabilidade (alta e muito alta) das medidas alcançadas com o uso da biofotogrametria. O procedimento clínico para a tomada da medida biofotogramétrica pode ser considerado mais simples em comparação com a técnica goniométrica, ficando o avaliador responsável apenas por palpar 
e demarcar os pontos anatômicos já definidos, sem preocuparse com qualquer alinhamento. $\mathrm{O}$ restante do procedimento torna-se puramente técnico, desdobrando-se com o registro fotográfico do ângulo avaliado e posterior análise com o uso de uma ferramenta digital $[3,22]$.

Desta forma, o procedimento para biofotogrametria demonstrou-se bastante prático para os avaliadores que puderam, assim, melhor aplicar seus conhecimentos e aproveitar de sua experiência em avaliação de amplitude de movimento. A grande maioria das pesquisas que utiliza esta técnica demonstrou uma confiabilidade variando de alta a muito alta, independente do período entre as avaliaçóes $[27,28]$.

Este fato pode apresentar uma importância clínica significante em cenários de prática fisioterapêutica, na qual deve ser necessária a tomada de várias medidas de amplitude em um mesmo momento, ou ainda, pode orientar a reproduçáo de pesquisas que venham a utilizar este procedimento para a medida de amplitude articular.

Em relação à objetividade, os resultados da biofotogrametria continuam sendo mais expressivos do que os da goniometria, indicando uma maior concordância dos resultados entre os avaliadores, quando estes utilizaram a biofotogrametria como técnica de medição da amplitude.

Venturini et al. [1] também não observou resultados expressivos para objetividade das avaliaçóes de amplitude ativa de tornozelo com o uso do goniômetro. Coelho [22] utilizando a biofotogrametria como técnica de avaliação da amplitude para articulaçáo do joelho, demonstrou boa concordância entre os valores obtidos por diferentes avaliadores. Contudo não foram encontrados estudos que comparassem os valores de objetividade entre a técnica goniométrica e biofotogramétrica, para uma mesma articulação.

Quanto à ausência de correlação das medidas destas duas técnicas de mensuração com a medida radiológica, este fato encontra-se em concordância com o ponto de vista de Norkin et al. [4] e Venturini et al. [12], que afirmam que apesar de já existirem diversos instrumentos para a medida do movimento articular, estes não devem ser utilizados de forma aleatória, nem tampouco permitem comparaçóes entre suas medidas. É notória a diferença entre os procedimentos destes instrumentos e técnicas de medidas, que se baseiam, muitas das vezes, em princípios e referências distintas.

Fish et al. [25], comparando avaliações goniométricas de uma amplitude fixa da articulação do cotovelo com avaliaçóes biofotogramétricas da mesma posição articular, encontraram diferenças estatísticas significantes nos valores obtidos.

Contudo, Coelho [22] percebeu uma forte correlação para medidas de posicionamento estático de joelhos de indivíduos sadios, entre a biofotogrametria e a goniometria radiológica. Este autor estabeleceu como um dos critérios de avaliação a exclusiva responsabilidade do avaliador pelas etapas das tomadas de medida de amplitude, que incluía desde a palpação dos pontos anatômicos até o posicionamento para o registro radiográfico.
A ausência desse mesmo critério, através da participação do técnico em radiologia no presente estudo, pode então ter sido responsável pela pouca correlação entre os valores da biofotogrametria e da goniometria radiológica.

Vale ainda ressaltar que esta forte correlação observada no estudo de Coelho [22], pode estar relacionada à confirmação da localização dos pontos anatômicos palpados pelos avaliadores, através da imagem radiográfica, definindo esta confirmação como mais um critério para o bom emprego da técnica biofotogramétrica.

\section{Conclusão}

O presente estudo demonstrou, portanto, baixa e moderada confiabilidade para os resultados com o uso do goniômetro, ao contrário dos valores observados para as medidas com o uso da biofotogrametria, que apresentam nível alto e muito alto de confiabilidade, estando estes valores em concordância com outros trabalhos já publicados nesta linha de estudo.

Em relação à objetividade, os resultados indicam uma maior concordância entre os avaliadores, quando estes utilizaram a biofotogrametria como técnica de mediçáo da amplitude. Contudo, carecem estudos que comparem o grau de objetividade entre estas duas técnicas. Sugere-se, então, que novos estudos sejam realizados, comparando-se a objetividade entre estas duas técnicas, afim de que se defina, entre elas, aquela que possui a maior consistência dos resultados quando as medidas forem realizadas por diferentes avaliadores.

Percebeu-se ainda baixa correlação entre os valores das medidas de ângulo colhidas com o uso do instrumento goniômetro e da técnica biofotogramétrica, em comparação aos valores encontrados como o uso da goniometria radiológica, para um mesmo ângulo.

É possível que esta baixa correlação encontrada entre as técnicas de medida de amplitude articular esteja relacionada à interferência de outro profissional, durante a tomada de medida radiológica, ou ainda que estes resultados apenas reforcem o que já foi afirmado por outros autores: de que técnicas diferentes de medida de amplitude articular não permitem comparação entre seus resultados.

\section{Referências}

1. Venturini C, Ituassú NT, Teixeira LM, Deus CVO. Confiabilidade intra e interexaminadores de dois métodos de medida de amplitude ativa de dorsiflexão do tornozelo em indivíduos saudáveis. Rev Bras Fisioter 2006;10(4):407-11.

2. Batista LH, Camargo PR, Aiello GV, Oishi J, Salvini TF. Avaliação da amplitude articular do joelho: correlação entre as medidas realizadas com o goniômetro universal e no dinamômetro isocinético. Rev Bras Fisioter 2006;10(2):193-98.

3. Amorim DA, Ribeiro EM, Cordeiro GG, Silva MAS. O Programa Autocad $2000^{\circ}$ como forma de medida angular para articulaçóes [TCC]. Itaúna: Universidade de Itaúna; 2005. 
4. Norkin CC, White DJ. Medida do movimento articular: manual de goniometria. 2a ed. Porto Alegre: Artes Médicas; 1997. p. 34-95.

5. Monteiro GA. Avaliação da flexibilidade: manual de utilização do flexímetro sanny. 1a ed. São Bernardo do Campo: American Medica do Brasil; 2005. p. 4-18.

6. Manal K, Chang C, Hamill J, Stanhope S. A three-dimensional data visualization technique for reporting movement pattern deviations. J Biomech 2005;38:2151-56.

7. Fua P, Gruen A, Plänkers R, D’Apuzzo N, Thalmannl D. Human body modeling and motion analysis from video sequences. Hakodate: International Archives of Photogrammetry and Remote Sensing; 1998. p. 866-73.

8. Morphett AL. The use of electromagnetic tracking technology for measurement of passive cervical range of motion: a pilot study. J Manipulative Physiol Ther 2003;26(3):152-59.

9. Sprigle S, Flinn N, Wootten M, McCorry S. Development and testing of a pelvic goniometer designed to measure pelvic tilt and hip flexion. Clin Biomech 2003;18:462-65.

10. Fernandes Filho J. A prática da avaliação física. 1a. ed. Rio de Janeiro: Shape; 1999. p. 129-153.

11. Norton K, Olds T. Antropométrica. 1a ed. Porto Alegre: Artmed; 2005. p. 39-104.

12. Venturini C, André A, Aguilar BP, Giacomelli B. Confiabilidade de dois métodos de avaliaçáo da amplitude de movimento ativa de dorsiflexão do tornozelo em indivíduos saudáveis. Acta Fisiatr 2006;13(1):39-43.

13. Polanowski DW. A validade e a reprodutibilidade de flexímetro sanny. In: Anais do $18^{\circ}$ Congresso Internacional de Educação Física - FIEP, Foz do Iguaçu, Jan. 2003.

14. Baraúna MA, Ricieri D. Biofotogrametria: recurso diagnóstico do fisioterapeuta. O Coffito 2002;3:7-11.

15. Baraúna MA, Barbosa SEM, Canto RST, Silva RAV, Silva CDC, Baraúana KMP. Estudo do equilíbrio estático de idosos e sua correlação com quedas. Fisioter Bras 2004;5(2):136-41.

16. Portney LG, Watkins MP. Reliability. In: Portney LG, Watkins M.P. Foundations of clinical research applications to practice. New Jersey: Prentice-Hall 2000; p. 61-75.
17. Rothstein JM, Miller PJ, Roettger RF. Goniometric reliability in a clinical setting: elbow and knee measurements. Phys Ther 1983;63:1611-15.

18. Batterham AM, George K. Reliability in evidence-based clinical practice: a primer for allied health professionals. Phys Ther Sport 2003;4:122-28.

19. Martelli Filho JA, Maltagliati LA, Trevisan F, Gil CTLA. Novo método estatístico para análise da reprodutibilidade. Rev Dent Press Ortodon Ortop Facial 2005;10(5):122-29.

20. Dantas EHM, Carvalho JLT, Fonseca RM. O protocolo LABIFIE de goniometria. Revista Treinamento Desportivo 1997;2(3):21-34.

21. Field D. Anatomia palpatória. 2a . ed. São Paulo: Manole; 2001. p. 26.

22. Coelho RR. Validade e reprodutibilidade de um protocolo de avaliação de amplitude de movimento da articulação do joelho pela biofotogrametria computadorizada [dissertação]. Rio de Janeiro: Universidade Castelo Branco; 2006.

23. Tiziani C, Vicola FRR. Avaliação do movimento de flexo-extensão do punho com o uso da biofotogrametria [online]. [citado 2007 Jul 28]. Disponível em URL: http://www.mao2007.com. br/ger encial/mostra_resumo.asp?traid=1 \&insid=95>

24. Alphons MPM, Baak MAV, Vrencken JGPM, Wijnen JAG. Verstappen, FTJ. Variability and reliability of joint measurements. Am J Sports Med 1990;18:58-63.

25. Fish DR, Wingate L. Sources of goniometric error at the elbow. Phys Ther 1985;65(11)1666-70.

26. Ueda TK. Avaliação qualitativa dos trabalhos de conclusão de curso do curso de fisioterapia da universidade estadual do oeste do Paraná [TCC]. Foz de Iguaçu: Universidade Estadual do Oeste do Paraná; 2005.

27. Coelho RR, Coelho GHL, Silve EM, Dantas EHM. Confiabilidade para a avaliação da amplitude de movimento ativa máxima de extensão do joelho pela biofotogrametria. Anais XVI COBRAF, São Paulo; 2005.

28. Farber DC, Deorio JK, Steel MW. Goniometric versus computerized angle measurement in assessing hallux valgus. Foot Ankle Int 2005;26(3):234-38. 